\title{
Study on the Post Evaluation of the Chengshan Jiao Routeing system Based on Entropy Method
}

\author{
Ze-ning Meng ${ }^{1}$, Zhong-zhou Fan ${ }^{2,}$ and Xue-dong Yang ${ }^{3}$ \\ ${ }^{1,2,3}$ China, Dalian, Dalian Maritime University: Navigation College
}

\begin{abstract}
Keywords: Chengshan Jiao Waters, Ship Routeing Scheme, Index Weighting Method, Entropy Method, Combination Weighting Method.
\end{abstract}

\begin{abstract}
In order to do further study of newly amended Chengshan Jiao Routeing system, it's necessary to conduct a post evaluation. Based on the result of questionnaires, this paper collected and quantified crew's and maritime experts' assessments on the Chengshan Jiao Routeing system's safety indexes and then constructed a safety index weighting model by applying Entropy Method, Analytic Hierarchy Process and Combination Weighting Method, the calculated results were very much in line with the actual situation. This model shows a strong applicability and has a relatively simple calculation process. This research aims to provide a highly operational assessment method for post evaluation of traffic separation scheme.
\end{abstract}

\section{Introduction}

Safety assessment of routeing system is a vital and sensitive process in post evaluation. In this kind of assessment, we always encounter difficulties not only in performing criteria and indexes, but also in designing a comprehensive and effective model to assign weights to these indexes. Previous models of weighting mainly focused on single method, for example, Fuzzy Analytic Hierarchy Process (FAHP) has been used to evaluate shipping routes in South China Sea[1] ;entropy method has been used for assessment of routeing system[2]etc. Although these models can solve problems to some extent, we still can't skip the limitation and one-sidedness of the method itself, which may cause errors in our evaluation. To minimize these errors, this paper casts gaze on constructing a new hybrid model which integrates Entropy Method, Analytic Hierarchy Process (AHP)[3-5] and Combination Weighting Method[6-8] in collecting and quantifying crew's and maritime experts' assessments on the Chengshan Jiao Routeing system's safety indexes by questionnaires. Ultimately, this paper, aimed at verifying the practicability of this model, is employed to assess the safety of newly amended Chengshan Jiao Traffic Separation Scheme(TSS). The result shows a great concordance with the current situation.

\section{Safety Index}

According to general rules and experts' opinion, as well as natural and sailing situation in Chengshan Jiao area combined with various factors that may affect the establishment of routeing system[9-10], this paper comes up with an index system on safety evaluation of routeing system in Chengshan Jiao area as follows:

\section{Weighting Model}

Weight is a crucial indicator to tell the imparities between different indexes, in other words, it shows the level how these indexes influence the whole system. In order to minimize the adverse effects caused by single method, this paper, creatively integrated Entropy Method, Analytic Hierarchy Process (AHP) and Combination Weighting Method together and design a new model for safety evaluation of TSS. 
Table1. Safety index

\begin{tabular}{cl}
\hline No & Safety index \\
\hline 1 & $\begin{array}{l}\text { General result of setting up the outer traffic separation schemes which divides territorial waters into } \\
\text { North-South and East-West Traffic flows } \\
2\end{array}$ \\
Whether the second alert zone in the outer traffic separation schemes can simplify or relieve the \\
crossing situation in VTS jurisdictions \\
The effect on safety of sailing caused by width of separation zone in both inner and outer traffic \\
separation schemes which is more than 2.5 nautical miles \\
The effect on safety of sailing caused by width of traffic lane in outer traffic separation schemes \\
which is 2 nautical miles \\
The effect on safety of sailing caused by width of separation zone in outer traffic separation schemes \\
which is 2 nautical miles \\
The effect on safety of sailing caused by outer alter zone which covers a region 10 nautical miles \\
long and 5 nautical miles wide \\
Whether the suggestion that passenger ship; high speed ship; large size vessel and dangerous cargo \\
vessels sail in the outer traffic separation schemes has improved safety in general.
\end{tabular}

\section{Method}

Entropy Method. Entropy is a parameter that manifest the uncertainty of the system in information theory. The more information a system has, the less uncertainty it will be, thus, the entropy will get smaller and vice versa. Entropy Method is exactly using the aforementioned principle. Specific steps are illustrated as follows:

Step 1:Each unstandardized factor in the evaluation matrix $X=(x i j) m \times n$ has its own characteristics. To compare them directly, a new standardized matrix $\mathrm{Y}=(\mathrm{yij}) \mathrm{m} \times$ nshould be obtained and then be normalized to get a matrix represented as:

$$
P_{i j}=y_{i j} / \prod_{i=1}^{m} y_{i j}(1 \leq i \leq m, 1 \leq j \leq n)
$$

Step 2: Calculate the entropy of the No. $\mathrm{j}$ index by the following equation:

$$
e_{j}=-k \cdot \sum_{i=1}^{m} p_{i j} \ln p_{i j}(1 \leq j \leq n)
$$

where $\mathrm{k}>0$,ej $>0$;

Step 3: When it comes to the influence on evaluation caused by the coefficient of the No. $j$ index, we can clearly realize that it goes to entirely opposite side, that's to say, if the entropy of the No. $\mathrm{j}$ index gets smaller, the coefficient will be bigger, thus it provides more references to assessment. As a result, a difference coefficient gj should be defined as:

$$
g_{j}=1-e_{j}(1 \leq j \leq n)
$$

Step 4: Calculate the weight of the No. $\mathrm{j}$ index by the following equation:

$$
\omega_{j}^{\prime}=g_{j} / \sum_{j=1}^{n} g_{j}
$$

Analytic Hierarchy Process (AHP). Analytic Hierarchy Process is an efficient method to convert complex decision-making system into a hierarchical structure for the purpose of comparing different factors to get a quantitative gist for evaluation. Specific steps are as follows:

Step 1: Contribute a judgment matrix as shown below: 
Table 2. Judgment matrix

\begin{tabular}{ccccc}
\hline & $\mathrm{Q}_{1}$ & $\mathrm{Q}_{2}$ & --- & $\mathrm{Q}_{\mathrm{n}}$ \\
\hline $\mathrm{Q}_{1}$ & $\mathrm{q}_{11}$ & $\mathrm{q}_{12}$ & --- & $\mathrm{q}_{1 \mathrm{n}}$ \\
$\mathrm{Q}_{2}$ & $\mathrm{q}_{21}$ & $\mathrm{q}_{22}$ & --- & $\mathrm{q}_{2 \mathrm{n}}$ \\
$\vdots$ & $\vdots$ & $\vdots$ & & $\vdots$ \\
$\mathrm{Q}_{\mathrm{n}}$ & $\mathrm{q}_{\mathrm{n} 1}$ & $\mathrm{q}_{\mathrm{n} 2}$ & --- & $\mathrm{q}_{\mathrm{nn}}$ \\
\hline
\end{tabular}

Where the judgment rules of specific importance should be represented as:

Coordinate importance donated as 1 ;

Slight importance donated as 3 , on the contrary as $1 / 3$;

Obvious importance donated as 5 , on the contrary as $1 / 5$;

Extraordinary importance donated as 7 , on the contrary as $1 / 7$;

Extreme importance donated as 9, on the contrary as 1/9;

$2,4,6,8$ can be used to express median importance.

Step 2: Get the consistent matrix by the following equation:

$$
\mathrm{c}_{i j}=\sqrt[n]{\prod_{k=1}^{n} q_{i k} \cdot q_{k j}}
$$

Where $c i i=1, c i j=1 / c j i, c i j=c i k * c k j$.And then, define dj as:

$$
d_{j}=\sqrt[n]{\prod_{k=1}^{n} c_{j k}}
$$

Step 3: Express the weight of indexes with the equation below:

$$
\omega^{\prime \prime}=d_{j} / \sum_{k=1}^{n} d_{k}
$$

Combination Weighting Method. Firstly, we should perform a consistency checks with Kendall coefficient before making use of the aforementioned methods based on the following steps:

Step 1: Assuming that we weight $\mathrm{n}$ indexes with $\mathrm{m}$ method, thus, we will get an ordinal matrix which contains the results we got from the aforementioned methods as:

$$
Y=\left[\begin{array}{cccc}
y_{11} & y_{12} & \cdots & y_{1 m} \\
y_{21} & y_{22} & \cdots & y_{2 m} \\
\vdots & \vdots & & \vdots \\
y_{n 1} & y_{n 2} & \cdots & y_{n m}
\end{array}\right]
$$

Where yij represent the rank of $\mathrm{i}$ index with $\mathrm{j}$ method, $y i j \in \mathrm{N}, 1 \leq y \mathrm{ij} \leq \mathrm{n}$.

Step 2: If $\mathrm{m}$ methods show inconsistency, we use $\mathrm{H} 0$ to represent it. And $\mathrm{H} 1$ to express consistency.

Step 3: Calculate test statistic and verify the assumption by the followings:

(1) When $n \leq 7$, the test statistic can be calculated as:

$$
s=\sum_{i=1}^{n} R_{i}^{2}-\frac{1}{n}\left(\sum_{i=1}^{n} R_{i}\right)^{2}
$$

Where, $s$ is Kendall coefficient. If significant level $\alpha$ has been given, the threshold s $\alpha$ can be easily found in the table of $\mathrm{s}$.

(2) When $\mathrm{n} \geq 7$, the test statistic can be calculated as: 


$$
i^{2}=m(n-1) T
$$

Where $T=\frac{12 \sum_{i=1}^{n} R_{i}^{2}}{m^{2} n\left(n^{2}-1\right)}-\frac{3(n+1)}{n-1}, \quad R_{i}=\sum_{j=1}^{m} y_{i j}, \mathrm{i} 2$ is subject to the i2 distribution with $\mathrm{n}-1$ degrees of freedom.

Step 4: Look through the chi-square distribution table according to $\mathrm{T}$ and $\mathrm{n}-1$ to get the threshold $i_{x^{2}}^{2}$.If $i^{2}>i_{x^{2}}^{2}$, we can come to the conclusion that m methods show consistency in a significance level of T, that is, refuse $\mathrm{H} 0$ and accept $\mathrm{H} 1$.

Then, once it passes the consistency checks, we can combine $\omega_{j}^{\prime}$ and $\omega_{j}^{\prime \prime}$ by the following equation:

$$
\omega_{j}=k_{1} \omega_{j}^{\prime}+k_{2} \omega_{j}^{\prime \prime}
$$

Where $\mathrm{k} 1$ and $\mathrm{k} 2$ are undetermined constants, $\mathrm{k} 1+\mathrm{k} 2=1, \mathrm{k} 1>0, \mathrm{k} 2>0$

Calculate $\mathrm{k} 1$ and $\mathrm{k} 2$ with Lagrange extremum principle at the precondition of $\mathrm{k} 12+\mathrm{k} 22=1$

$$
\begin{gathered}
k_{1}=\sum_{i=1}^{m} \sum_{j=1}^{n} \omega_{j}^{\prime \prime} r_{i j} / \sqrt{\left(\sum_{i=1}^{m} \sum_{j=1}^{n} \omega_{j}^{\prime} r_{i j}\right)^{2}+\left(\sum_{i=1}^{m} \sum_{j=1}^{n} \omega_{j}^{\prime \prime} r_{i j}\right)^{2}} \\
k_{2}=\sum_{i=1}^{m} \sum_{j=1}^{n} \omega_{j}^{\prime} r_{i j} / \sqrt{\left(\sum_{i=1}^{m} \sum_{j=1}^{n} \omega_{j}^{\prime} r_{i j}\right)^{2}+\left(\sum_{i=1}^{m} \sum_{j=1}^{n} \omega_{j}^{\prime \prime} r_{i j}\right)^{2}}
\end{gathered}
$$

In this way, the result can reflect the biggest difference between $\mathrm{k} 1$ and $\mathrm{k} 2$, in other words, the value of Eq.(14) will reach the highest point.

$$
\sum_{i=1}^{m} \chi_{i}=\sum_{i=1}^{m} \sum_{j=1}^{n}\left(k_{1} \omega_{j}^{\prime}+k_{2} \omega_{j}^{\prime \prime}\right) r_{i j}
$$

$\mathrm{k} 1$ and $\mathrm{k} 2$ should be normalized to ensure the condition in Eq. (11) as $k_{1}^{\prime}=k_{1} / k_{1}+k_{2}$, $k_{2}^{\prime}=k_{2} / k_{1}+k_{2}$. Then we can get final weight by $\omega_{j}=k_{1}^{\prime} \omega_{j}^{\prime}+k_{2}^{\prime} \omega_{j}^{\prime \prime}$.

\section{Process of Model}

From what has been discussed above, the process of whole model can be summarized as follows:

(1) Create a matrix A to describe the relationship between the value of indexes and the object.

(2) Apply dimensionless method and normalization to A to get a new matrix P

(3) Calculate entropy E of No.j index

(4) Calculate difference coefficientg $\mathrm{j}_{\mathrm{j}}$ of No.j index

(5) Calculate entropy weight of No.j index

(6) Calculate combined weight of No.j index

(7) Calculate evaluation value by the following equation

$$
Z_{i}(\omega)=\sum_{j=1}^{m} r_{i j} \omega_{j}
$$




\section{Preponderance of This Model}

Compared with the traditional weighting model, the most significant advantage of this model is that the sidedness and limitation of the single weighting method can be avoided, that is, this model can balance both the objectivity of Entropy Method and the subjectivity of AHP, meanwhile,strive to reduce subjective randomness on the premise of the preference for specific attribute. These preponderances can increase the accuracy of assessment to some extent, thus this model can provide a reliable choice for safety assessment of TSS.

\section{Safety Assessment of Newly Amended Chengshan Jiao Routeing System}

In order to prove the feasibility and accuracy of the weighting model, we applied it to the newly amended Chengshan Jiao Routeing system. The results were in accordance with the actual situation.

\section{Assign a Score to The Result of Questionnaires}

Assuming that the median of score for tentative scheme is 3, the improvement effect in questionnaire can be assigned as 1 to 5 refer to different actual result. Multiply the result extracted from questionnaire by different score, then, divide it by the copies of questionnaire, we can get the following result:

Table 3. Safety index value

\begin{tabular}{ccc}
\hline Safety index sequence & Current version & Tentative version \\
\hline 1 & 4.129 & 3.000 \\
2 & 4.236 & 3.000 \\
3 & 3.805 & 3.000 \\
4 & 3.916 & 3.000 \\
5 & 4.008 & 3.000 \\
6 & 3.928 & 3.000 \\
\hline
\end{tabular}

\section{Weight}

Entropy Weight. Entropy weight can be calculated as follows:

(1) Normalize the data in Tab.3 by Eq.(1) to get matrix P:

(2) Calculate entropy Ej of No. j indexby Eq. (2):

$$
P=\left[\begin{array}{llllll}
0.483 & 0.459 & 0.484 & 0.496 & 0.482 & 0.493 \\
0.517 & 0.541 & 0.516 & 0.504 & 0.518 & 0.507
\end{array}\right]
$$

$$
E_{j}=\left[\begin{array}{llllll}
0.996 & 0.998 & 0.983 & 0.997 & 0.991 & 0.992
\end{array}\right]
$$

(3) Calculate weigh vector by Eq. (3) and Eq. (4):

$$
\omega^{\prime}=\left[\begin{array}{llllll}
0.152 & 0.108 & 0.329 & 0.146 & 0.162 & 0.103
\end{array}\right]
$$

AHP Weight. AHP weight can be calculated as follows:

(1) Construct a decision matrix Q according to the index system of safety evaluation, along with the analysis of questionnaire.

(2) Get consistent matrix C by Eq. (5)

$$
Q=\left[\begin{array}{cccccc}
1 & 3 & 1 / 2 & 1 / 2 & 2 & 2 \\
1 / 3 & 1 & 1 / 5 & 1 / 5 & 1 / 2 & 1 / 3 \\
2 & 5 & 1 & 2 & 3 & 2 \\
2 & 5 & 1 / 2 & 1 & 3 & 2 \\
1 / 2 & 2 & 1 / 3 & 1 / 3 & 1 & 1 / 2 \\
1 / 2 & 3 & 1 / 2 & 1 / 2 & 2 & 1
\end{array}\right]
$$


(3) Get matrix Dj by Eq. (6)

$$
C=\left[\begin{array}{llllll}
1.000 & 3.324 & 0.541 & 0.681 & 1.944 & 1.260 \\
0.301 & 1.000 & 0.163 & 0.205 & 0.585 & 0.379 \\
1.850 & 6.148 & 1.000 & 1.260 & 3.595 & 2.330 \\
1.468 & 4.880 & 0.794 & 1.000 & 2.854 & 1.849 \\
0.514 & 1.710 & 0.278 & 0.350 & 1.000 & 0.648 \\
0.794 & 2.639 & 0.429 & 0.541 & 1.543 & 1.000
\end{array}\right]
$$

$$
D_{j}=\left[\begin{array}{llllll}
1.201 & 0.361 & 2.221 & 1.763 & 0.617 & 0.953
\end{array}\right]
$$

(4)Calculate weight $\omega^{\prime \prime}$ by Eq. (7)

$$
\omega^{\prime \prime}=\left[\begin{array}{llllll}
0.169 & 0.050 & 0.312 & 0.248 & 0.087 & 0.134
\end{array}\right]
$$

Combined Weight. Combined weight can be calculated as follows:

(1) Make a consistency inspection in advance, sort the weight we have calculated above to get ordinal matrix A

$$
A=\left[\begin{array}{ll}
3 & 4 \\
5 & 1 \\
1 & 2 \\
2 & 3 \\
6 & 6 \\
4 & 5
\end{array}\right]
$$

(2) Based on $\mathrm{s}=9.6$ (calculated by Eq. (9)) and the given significant level $\mathrm{T}=0.6, \quad i_{x^{2}}^{2}=6.432$ can be searched from the chi-square distribution table, so $i^{2}>i_{x^{2}}^{2}$, thus, we will refuse H0 and accept $\mathrm{H} 1$, entropy method and AHP have consistence at the significant level $\mathrm{T}=0.6$, the weight calculated above can be combined.

(3) Utilize the Lagrange extremum principle, we can get that $\mathrm{k} 1=0.709, \mathrm{k} 2=0.705$, normalize them to get $k_{1}^{\prime}=0.499, k_{2}^{\prime}=0.501$. Then the combined weight should be:

$$
\omega=\left[\begin{array}{llllll}
0.138 & 0.070 & 0.382 & 0.221 & 0.063 & 0.126
\end{array}\right]
$$

\section{Safety Assessment}

According to Eq. (15), the evaluation value of current and tentative version can be represented respectively as:

$$
Z=\left[\begin{array}{ll}
4.29 & 3
\end{array}\right]
$$

That is, $Z 1>Z 2$. In consideration of extremum is 5 , the result explicitly shows that the safety of current amendment has been improved greatly.

\section{Conclusion}

This paper for purpose of quantifying the safety indexes roundly and reasonably and improving the veracity of assessment, gathered objective evaluations to safety indexes of the newly amended Chengshan Jiao Routeing system and constructed a new hybrid model which integrates Entropy Method, Analytic Hierarchy Process (AHP) and Combination Weighting Method. After apply it to the safety assessment of the newly amended Chengshan Jiao Routeing system, the result shows that the safety of newly amended TSS had a significant promotion of safety and this result was very much in line with the actual situation that there's nocollision accident in this area in nearly a year after this amendment is applied. This can also be regarded as a proof to certify the suitability of this model. The model produced by this paper will provide guidance for safety assessment of other routeing systems. 


\section{References}

[1] Jia-sheng Wang, Man-chun Li,Yong-xue Liu,ect.Safety assessment of shipping routes in the South China Sea based on the fuzzy analytic hierarchy process, J.Safety Science,2014,62:46-57.

[2] Zhang Hao, Yu Bin, Xiao Ying-jie, ect. Alternative design evaluation of ship's routeing system,J. Journal of Shanghai Maritime University,2010,31(3). (in Chinese)

[3] WANG Jing, ZHANG Jin-suo. Comparing Several Methods of Assuring Wight Vector In Synthetical Evaluation,J.Journal of Hebei University of Technology,2001,30(2):52-57.(in Chinese)

[4] FAN Zhong-zhou.Research on the Optimizedmethod of Ship Routeing Scheme,D.Dalian:Dalian Maritime University,2013.(in Chinese)

[5] FANG Xiang-lin, WANG Feng-chen, WU Zhao-lin.The Evaluating Method of Vessel Traffic Safety Index Method,J.Journal of Dalian Marine College,1992,18(4):337 341.(in Chinese)

[6] ZENG Xian-bao.New Probe on Combinatorial valuation method,J. Forecasting, 1997,16(5):69-72.(in Chinese)

[7] CHEN Wei, XIA Jian-hua. An Optimal Weights Combination Method Considering both Subjective and Objective Weight Information, J.Mathematics in Practice and Theory,2007,37(1): 17-22.(in Chinese)

[8] WANG Ming-tao. A Comprehensive Analysis Method on Determining The Coefficients in Multi-index Evaluation, J.Journal of systems engineering,1999,17(2):57-61.(in Chinese)

[9] Maritime Safety Administration in Weihai. Statistical analysis ontraffic flow in the east of Chengshan Jiao area, R.

[10] WU Jian-jun.Set-Pair Comprehensive Evaluation on the Navigation Environment of Traffic Separation Schemes Waters, D.Dalian: Dalian Maritime University, 2010.(in Chinese) 